\title{
Screening, isolation, and molecular characterization of putative fragrance-related transcripts from Vanda Mimi Palmer.
}

\begin{abstract}
The aims of this study were to isolate and characterize putative fragrance-related cDNAs from the floral cDNA library of Vanda Mimi Palmer, an orchid hybrid that has won several international awards for its sweet fragrance. A total of 1,000,000 pfu were screened by hybridizing cDNA library plaques with fully open flower cDNA probe of Vanda Mimi Palmer representing all mRNAs expressed during daytime. The clones that gave positive signals were in vivo excised and PCR-amplified inserts were subjected to reverse-Northern analysis by hybridizing with cDNA probes of fully open flower of Vanda Mimi Palmer (fragrant orchid) and its bud or fully open flower of Vanda Tan Chay Yan (non-fragrant orchid) separately. The clones up-regulated in fully open flower stage of Vanda Mimi Palmer compared to its bud stage or fully open flower of Vanda Tan Chay Yan were sequenced. Sequence analyses showed the presence of eight putative fragrance-related cDNAs of which two were putative Vanda Mimi Palmer 4-(cytidine 5'-diphospho)-2-C-methyl-d-erythritol kinase (VMPCMEK) and Vanda Mimi Palmer cytochrome P450 protein (VMPCyP450). These two transcripts were selected for full-length cDNA isolation and expression analysis by real-time RT-PCR. The VMPCMEK transcript encodes a polypeptide of 400 amino acid residues, while the VMPCyP450 encodes 538 amino acid residues. Relative expression analysis of VMPCMEK and VMPCyP450 transcripts by real-time RT-PCR showed upregulated expressions in floral tissues compared to vegetative tissues, and both were found to be developmentally regulated.
\end{abstract}

Keyword: Vanda Mimi Palmer; Cytochrome P450 protein; Fragrance-related transcripts. 